\title{
A PREVENTATIVE APPROACH TO MALARIA: Some Preliminary Considerations
}

MALARIA. THIS DISEASE, SPREAD VIA MOSQUITOS, IS ESPECIALLY PROMINENT IN DEVELOPING COUNTRIES. ALTHOUGH WE HAVE COMEA LONG WAYINOUR KNOWLEDGE OF MALARIA AND ATTEMPTS TO FIND DRUGS TO COMBAT IT, THE ABILITY OF THE MALARIA PLASMODIUM TO ADAPT TO TREATMENT IS PROBLEMATIC. CHLOROQUINE AND DDT, TWO FORMERLY EFFECTIVE COMPOUNDS, HAVE ALREADY BEEN RENDERED USELESS. THIS MAKES PREVENTITIVEAPPROACHES, SUCH AS EDUCATION, ESPECIALLY I MPORTANT. 


\section{INTRODUCTION}

Epidemics like malaria strike on a large and seemingly uncontrollable scale. While it is often difficult to comprehend the notion of eradicating such widespread diseases, recognizing the connection between humans, nature, and the diseases themselves is an essential first step. Like many other infectious diseases, malaria has had devastating effects on both public health and the economy. Because of the incredible adaptability of the mosquito and its anthropophilic tendencies, methods for slowing the spread of the disease should not only include treatments with drugs and pesticides but also involve the education of those at risk as well as of those treating the disease. It is not enough to simply address the biological mechanisms of malaria, of the mosquitoes that spread it, or of its victims. Humans, mosquitoes, their respective natural and social environments, and the interaction among all of these factors must also be strongly considered.

\section{THE ORGANISM BEHIND IT ALL}

Malaria is caused by plasmodia (single-celled and usually microscopic) parasites. There are hundreds of species of Plasmodium parasites each of which infects specific vertebrate hosts. Among these, four particular species infect human beings: Plasmodium vivax ( $P$. vivax), Plasmodium malariae (P. malariae), Plasmodium ovale ( $P$. ovale), and Plasmodium falciparum (P. falciparum). Of these four species, Plasmodium falciparum is responsible for most deaths, whereas other species of the parasite, although not as fatal, cause high morbidity and affect national economies.

The less deadly species have an impact on economies for logical reasons: the presence of the vector in the population may not kill nearly as many people as P. falciparum does, but it does damage the health of the working population. Not being able to work means less production which discourages trade and business both locally and internationally.

\section{INSIDE THE HUMAN BODY}

The malaria parasite enters the human host when an infected mosquito takes her blood meal. The parasite inside the mosquito is transmitted into the human blood through her saliva, which she injects into the flesh as she sucks her meal. The parasite, as a sporozoite, reaches the liver where it invades liver cells. Inside the liver cells it changes into round forms called merozoites, which are released into the blood to invade red blood cells. Inside the red blood cells they multiply into more merozoites and are finally released.

The cyclical development and release of the parasite in blood cells causes the disease malaria. The falciparum malaria makes the infected red blood cell sticky, causing them aggregate to the endothelial wall of the blood vessels. This can block blood flow in small capillaries and can cause cerebral thrombosis, which essentially is the formation of a blood clot in an artery supplying the brain with blood. This gradually cuts off the blood supply to that particular area of the brain and causes a stroke, often leading to death. Sometimes, some parasites in humans can go on to transform in a sexual stage and become a gametocyte.

When a mosquito then feeds on the infested blood of the infected individual, the parasite enters the mosquito as a gametocyte. Gametocytes live inside the red blood cells of a mosquito and emerge from it. Here, sexual fertilization occurs, and the union of two gametes forms a zygote. The zygote transforms into a slug-like structure called an ookinete. The ookinete then egresses from a mass of blood called the bolus and crosses the mosquito's intestinal wall. It remains attached to the intestine and grows as a cyst, now becoming an oocyst. Inside the oocyst the parasite multiplies a thousand-fold of sporozoites. The sporozoite is the form into which the parasite develops to invade the salivary glands.

\section{Physical Symptoms}

The release of the parasite from the red blood cells coincides with the violent rises in body temperature during the fever attacks commonly seen in malaria. The body temper- 


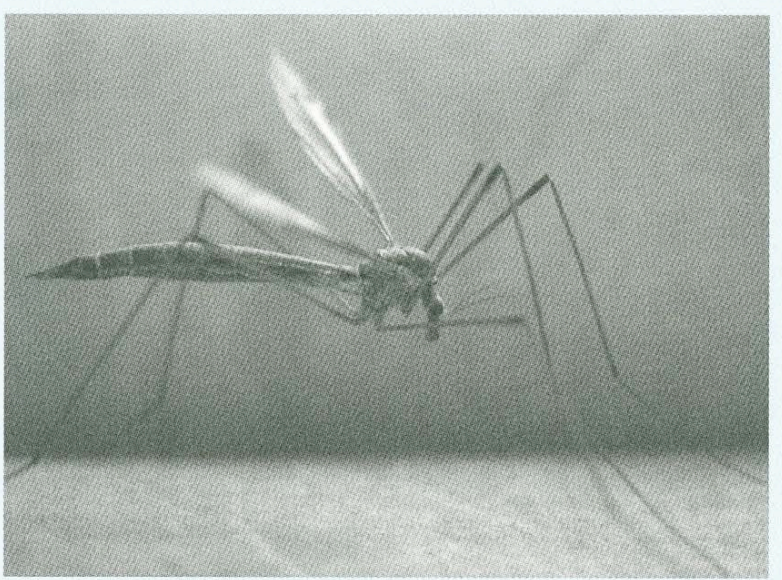

ature can reach an astonishing 106 degrees Fahrenheit.

However, this massive fever is preceded first by a series of other unmistakable symptoms. Andrew Spielman's book, Mosquito, gives a very vivid account of the body under attack by this debilitating disease:

Seven to fourteen days after an infecting bite, malaria begins with a chill that spreads like Jack Frost on a windowpane. The victim's skin becomes pallid, and shaking begins. Then the cold plunges to the body's core and deep tremors called rigors - come in waves. Though weakened by the disease, a man who feels the ice of malaria will shake with a violence that can propel an iron bed across the floor. Completely out of the victim's conscious control, the spasms are the body's vain attempt to generate the heat. Of course, the man, woman, or child who lies in the parasite's grip soon comes to understand that fever will inevitably follow the chill, and bring the next stage of suffering.

The fever from malaria...brings a sweat so profuse that doctors can almost see the droplets forming on the patient's forehead. They certainly see the rivulets that run down the tem. ples and onto the pillow. The bedclothes and sheets of the malaria victim become soaked with salty perspiration that is the by-product of the body's valiant effort to cool and protect the vital organs.
Death is usually unavoidable for those who are physically weakened by age or previous illnesses. The dying decay physically while their red blood cells, made sticky by the parasite, form into a plaque that clogs the vascular system and starves the brain. Most cases usually follow the common pattern of lethargy, delirium, and then coma. Unfortunately, those who have survived the initial episode of malaria are not necessarily cured of it; the progeny of the original parasites can remain in the body for months or years. They are usually held under control by the body's immune system, but any cause of decline of the immune function can trigger the disease to return in all its virulence.

\section{TRANSMITTANCE}

Malaria was originally thought to have originated in rancid marshes; hence mal aria is an Italian phrase meaning "bad air." Contrary to early understanding, however, it was actually a result of little creatures in the air which had been no more than an outdoor nuisance. In fact, many people did not realize that this minute insect was responsible for a worldwide epidemic.

Malaria is transmitted to humans by the bite of the female Anopheles mosquito, which in turn needs human blood to nurture her eggs. Anopheles mosquitoes mainly hunt for blood between dusk and dawn. Though some may feed at other times of the night, rarely are they around during the bright, sunny parts of the day. This is an immense benefit to the mosquitoes, especially in countries like Africa, because it gets cooler between dusk and dawn. People usually sit outside and rest or eat after a long day of work in the sun, making a buffet of human blood available to the mosquitoes. However, these insects are not only found in hot, dry climates like that of Africa because they can thrive anywhere from temperate to tropical climates, from Europe to South Africa. They can breed in any area with high humidity where there is warmth and a relatively permanent source of water. Distinguishing an uninfected mosquito from an infected mosquito cannot be done easily, ${ }^{i}$ but now there are molecular techniques available to detect malariainfected mosquitoes within a population. 


\section{A SPREADING THREAT}

Malaria has had an effect on human history since ancient times. In ancient China, men would arrange for the remarriage of their wives if they ever planned to travel to malarious areas; Alexander the Great is thought to have died in 323 B.C. as a victim of malaria; the city of Carthage was known to be plagued by malaria during the time of Christ; malaria probably defended Western Europe from falling into the hands of Genghis Khan. Despite its profound existence since the very first human beings, malaria was carried, undetectedly, by mosquitoes until the I 890 .

Today, approximately 4 I percent of the world's population, mostly those living in the world's poorest countries, is at risk of contracting malaria. ${ }^{i i}$ "Developed countries are likely to stay malaria-free," said Professor Andrew Haines of the London School of Hygiene and Tropical Medicine. Any outbreaks would be scattered cases, from insects unwittingly stowed away on airplanes. Countries currently most at risk are in the Third World or at the fringes where healthcare systems are weak. Some studies say malaria may reach higher altitudes in mountainous areas of developing countries. iii However, the malaria epidemic could easily become an international issue. While the improvement and success of many national economies is a result of globalization, the fading of geographical barriers introduces malaria vectors to vulnerable areas and drains those national economies.

When malaria hits, it leaves the region's economy damaged. Sick people are unable to work; crops and other goods are ruined and left abandoned for fear that they are swarming with the parasites and will affect the remaining healthy population. The community may be forced to evacuate the area and populate other regions originally free of malaria. Unfortunately, those displaced introduce the parasite to more unprotected people. Human carriers of the disease often show no symptoms, and when they move into previously malaria-free areas, the mosquitoes that bite them can pick up the parasite and go on to transmit it to other people. The chain of events can lead to a debilitating drop in the region's economy, which makes it difficult for the residents to afford the proper medication and treatment, which then leads to more sickness and death. The cycle continues as malaria spreads, ultimately becoming an international economic issue in this age of globalization.

Malaria is also spread to unprotected communities through the increasing popularity and ease of overseas travel. Infected mosquitoes may be introduced to new areas by being transported in planes or ships, or the parasite can be introduced to a new area via infected but asymptomatic returning travelers and foreign visitors. This is similar to how many of the immune African slaves unknowingly brought yellow fever to the West, or how the Europeans introduced foreign diseases that wiped out entire indigenous populations in the New World. It illustrates human means of transportation as an aide to the longer-distance journeys of the mosquito across borders and over oceans.

Malaria made its way to the United States through the importation and exportation of certain materials. These materials were then redistributed to other formerly non-malarious parts of the world, thus continuing the spread of malaria. The journey of Aedes albopictus (the Asian tiger mosquito) is an example of how a species of mosquito, though non-malarious, can come into the United States. Anopheles mosquitoes that carry malaria may have made their way to the United States in a similar manner.

The Asian tiger mosquito arrived in the U.S. in the late I970s because the U.S. began to import many used tires from Asia, where recapping worn tires and reusing them is illegal. However, reusing tires is not illegal in U.S. The tires were shipped to Houston, the tire recapping capital of the world, along with their unwelcome guests. Mosquito larvae were present in water that had pooled in these tires, while their eggs adhered to the inside walls of the tire. This type of mosquito usually breeds in water-filled cavities, like rot holes in trees. Thus, when humans began to save used tires, mosquitoes found the most ideally textured and the most perfectly simulated tree hole. Since it is difficult to 
empty used tires of the water inside them, they make the perfect mosquito maternity wards. Roughly four billion old tires are piled up on the American landscape today, with millions more being added every year. Due to the fact that many of the tires that arrive in Houston are redistributed worldwide, there is no stopping the spread of the Asian tiger mosquito.

Fortunately, malaria in the United States was only a close call, although that does not mean that malaria will not be able to strike again with worse consequences. The largest single factor that allowed the defeat of malaria in the United States seems to be the Tennessee Valley Authority (TVA), which designed every water project to limit mosquito breeding. Dams, irrigation networks, power systems, and drainage projects were built in the most malarious parts of the South. Where standing water could not be avoided, it was monitored for anopheline larvae, and larvicides, which are insecticides that float just on or under the film on the water's surface, were applied. These larvicides destroy anopheline larvae easily because an Anopheles mosquito feeds on material that is directly associated with the surface and breathes through two pores located at the end of its abdomen, which is kept in close contact with the water surface.

As shown by the TVA, civilization and the productive use of land can be beneficial not only to the health and wealth of the inhabitants, but also an effective way to keep the malaria epidemics at bay. However, other cases show that mosquitoes, no matter what the circumstances, malarial or not, constantly adapt to new environments. This poses an incredibly difficult challenge to overcoming malaria.

The introduction of artificial containers to the environment was a great benefit to the tree-hole mosquito. These insects prefer any small openings in which water can be collected. In our throwaway society, discarded food containers that accumulate near houses can serve as tree-hole replicas where a mature female mosquito can readily find sources of blood needed for egg production. Ultimately, people supply the mosquito with all she needs: artificial, water-collecting tree holes and a blood supply. Although this particular case does not involve the Anopheles species in particular, it indicates how mosquitoes have learned to survive among us.

In the early I880s, the French attempted a canal project to connect the Atlantic and Pacific. The failure of this project unlocked some of the mosquitoes' secrets:

The camps built to house the Panama Canal workers were probably the best in the tropics. But because mosquitoes were considered nothing more than a nuisance, screens were one luxury that was not indulged. In time, the barracks and even the hospitals built for construction workers became feeding areas for local mosquitoes. The French aided the insects' breeding as well, by decorating their compounds with lavish gardens where pottery rings filled with water were used to protect hundreds of trees from ants. These rings teemed with mosquito larvae. iv

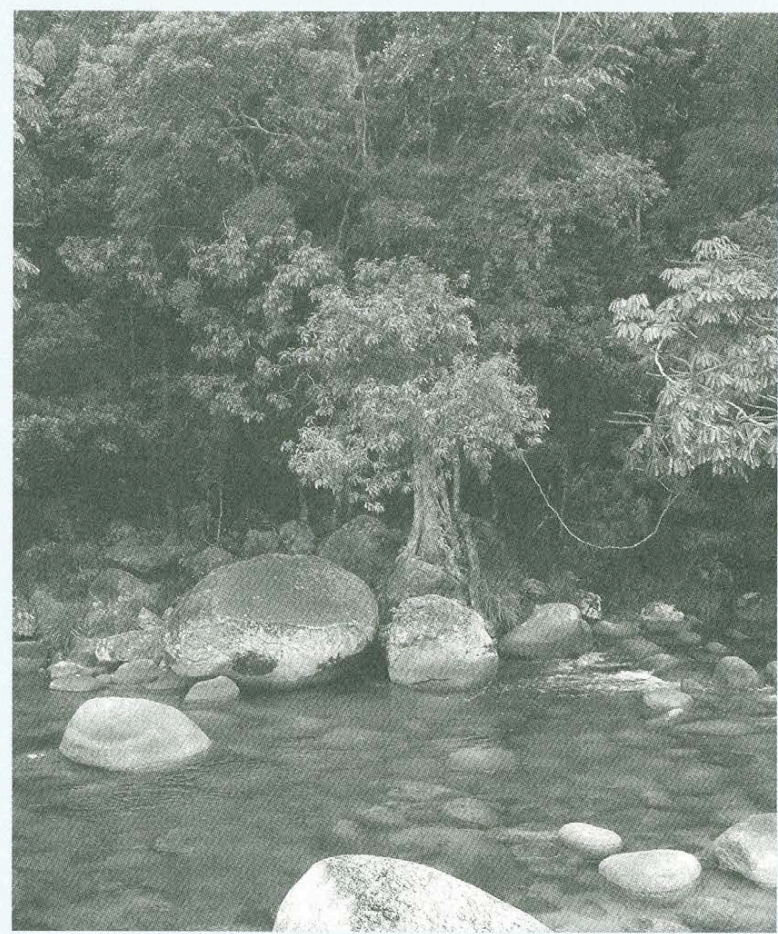


Another example of precautions to be considered in housing appears in parts of Africa. Andrew Spielman, a leading malariologist at the Harvard School of Public Health, explained in a conversation that maize fields around African homes serve as locations for mosquitoes to be closer to their human feed. Mosquitoes use the maize pollen as their food and lay their larvae in the mud, which is used to build the houses. Furthermore, there are usually mud ditches close to the homes for convenience. Many of these ditches are left open after the mud has been dug out, leaving ideal breeding sites for mosquitoes when they are filled with water. Clearly, it is important to assess the housing situations for places that are threatened by malarial mosquitoes for indirect relationships to malaria transmittance.

Futhermore, U.N. reports say rising temperatures linked to human burning of fossil fuels are likely to widen malaria's range in the tropics because mosquitoes and the malaria parasite they pass on (when sucking human blood) thrive best in hot, wet climates. Paul Reiter, professor of medical entomology at the Pasteur Institute in Paris, admits mosquitoes and plasmodia parasites reproduce faster in hot, damp climates but says that, on the other hand, higher temperatures might trigger floods that wash away stagnant pools in which malaria larva breed or spread deserts to dry up the waters. "More rainfall sometimes means more malaria, it sometimes means less," he said. All experts agree that there is slight risk that clouds of malaria-bearing mosquitoes will eventually make their way north to make the disease again endemic in nations from the United States to Russia. ${ }^{\vee}$

\section{FIGHTING MALARIA}

Although medicines were developed to mitigate the victim's suffering, the evolution of drug-resistant malaria parasites soon followed. The compound known as chloroquine was developed after World War II and was thought to be the ultimate cure for malaria - not only was it highly effective with fewer side effects, but it was also inexpensive, costing no more than a few cents compared to today's cost of approximately eight dollars a pill. vi Chloroquine's mirac- ulous abilities, however, were short-lived after its popularity led to widespread drug resistant malaria parasites, which were first seen in South American mosquitoes in I960 but later spread around the world.

After the creation of the controversial DDT, the United States was able to stay malaria-free, Europe eradicated malaria. Believing that DDT was the ultimate weapon for mass destruction of the mosquito, many other countries jumped on board with DDT; for a while, it was very effective. Australia began to spray their tire shipments, and other countries began to fumigate the cabins of aircrafts arriving from abroad, even with passengers in them. Because of DDT's reputation as the "end all, be all" solution, research grants for DDT disappeared overnight. There was no need for further research because it was assumed that the battle had been won.

Soon enough, the hype of DDT caused an unnecessary overuse of the chemical, allowing the mosquitoes to quickly build resistance. Moreover, Rachel Carson showed that, among many other hazards, DDT was found in mother's milk and could accumulate in babies, illustrating the chemical's ability to reach and harm the public. Consequently, the federal Food and Drug Administration announced that, "It's extremely likely that the potential hazard of DDT has been underestimated." vii Workers who handled the chemical developed health problems, and liver cancer was found in exposed fish. To make matters worse, malaria had begun its return.

Some recent developments in medicinal treatments include the use of a fungus fatal to the mosquito ${ }^{\text {viii }}$ and tests with the ancient Chinese plant Artemesia. However, as new drugs come and go as a consequence of the parasite's everincreasing resistance, and as there is a continuous chase to remain ahead of mosquitoes' resistance to man-made chemicals designed to kill them, it becomes increasingly imperative to stress the importance of making changes in our daily habits and in our surroundings as the most effective protection from malaria-transmitting mosquitoes. 


\section{CONCLUSION}

In some ways, it seems that the refinements modern society continually brings have worsened the malaria epidemic. Both the industrial progress of mankind and the ever-evolving nature of the mosquito make fighting malaria a difficult and exhausting battle. However, there is no doubt that our knowledge of malaria and the behavior of the mosquito carriers have progressed immensely since it was first observed.

Instead of pouring money into more and more pills and shots, it is now evident that investing in proper education about the mosquito and malaria can lead us to effective prevention methods. This is especially important to countries that do not have the medical resources or other proper means of protection. A simple change in habit or lifestyle can dramatically decrease the number of bites that an individual gets in a day. Most malaria vectors bite at night; some like A. gambiae generally bite predominantly indoor late at night, while others such as A. albimanus in Central America attack outdoors early in the evenings. The time of biting and whether it occurs indoor or outside are both epidemiologically important and relevant to control strategies. The on-going and uphill battle against malaria needs to utilize all viable methods, after the many previous failures in the search for a way to eradicate malaria.

\section{FURTHER STUDY}

In light of trying to present the power that anthropological and environmental factors have over infectious diseases, I would like this project to continue into an exploration of the cultural beliefs held by endemic regions that influence the population's response to the disease's presence. This includes the general attitude to Western medicine and treatment. Ultimately, the objective is to encourage Western medical practices to work with a region's culture and its traditions instead of blindly implementing their own methodologies. This will not only show respect for tradition, but will also be a more effective solution to the issue.
A friend mentioned an interesting belief that lies around the river Ganga (pronounced Ganges in the West) in India. The Ganga River is testable for mercury, arsenic, and dozens of other poisons, along with various parasites and bacteria. Some of this is due to the ritual of interring the dead in the river. Yet, if one asks a traditional, spiritual Indian about the river, he will insist that it is the purest water in the world. To them it is, after all, holy. ix

To the West, however, it is clearly unsanitary. And if the Ganga ever became a cause for an endemic disease like malaria, Westerners would react very differently than Indians would. From my observation, Westerners seem make it a priority to put the safety of people before cultural beliefs, whereas Indians may find prefer to endure the disease rather than to disregard tradition. I hope to ameliorate the situation without infringing on anyone's cultural beliefs, but it is questionable if this is completely possible.

\section{ENDNOTES}

i. Beier et al., I990

ii. Center for Disease Control

iii. Doyle, Alister. PlanetArk.com, July 29, 2004

iv. Spielman

v. Doyle

vi. Spielman

vii.Spielman

viii. New York Times article: June 10, 2005

ix. This is an anecdotal tribute to my friend Wesley Saavedr, although I am unsure where the story originated.

\section{REFERENCES}

Alzate, Alberto; Spielman, Andrew. "A Sustainable Antimalaria Strategy for a Region of Intense Transmission." Ig86(?)

Beier, et.al. "Malaria Transmission in Urban Sub-Saharan Africa." The American Society of Tropical Medicine and Hygiene 2003 , pp. $169-176$

Doyle, Allister. "Global Warming Effect on Malaria." 2005 , PlanetArk.com

Hoffman, Stephen L. Malaria Vaccine Development: A MultiImmune Response Approach. I996American Society for Microbiology, Washington D.C. 
Lane, Richard P., Crosskey, Roger W. Medical Insects and Arachnids.1993, Chapman \& Hall, Cambridge, UK.

Lehane, M.J. Biology of Blood-Sucking Insects. 199I Harper Collins Academic, London, UK.

Lipowsky, Renate, et.al. "Sociomedical Aspects of Malaria Control in Colombia." Social Science Medicine Magazine, Vol. 34, No. 6, pp. 625-637. 1992, Great Britain, UK.

Spielman, Andrew, D'Antonio, Michael. Mosquito: The Story of Man's Deadliest Foe. 200 I Hyperion, New York, New York.

Yamin, Gavey. "Malaria Drug Politics." May 8, 2000.

EssentialDrugs.org World Health Organization (www.WHO.int)

Center for Disease Control (www.cdc.gov)

Net Doctor (www.netdoctor.co.uk)

www.RaintreeNutrition.com, 2005

www.MalariaFoundation.com. May 17, 1998

www.SeniorHealth.com

www.CancerWEB.com. Online Medical Dictionary, 2004.

New York Times article, June 10, 2005 